\title{
Analysis on the Conversion Mechanism of Electromagnetic Energy to Continuous Movement
}

\author{
Dan Xia ${ }^{\mathrm{a}}$, Dianyou Song and Zhiyong Wang \\ School of Electronic Information Engineering, Tianjin Key Laboratory of Film Electronic and Communication Device, Tianjin \\ University of Technology, Tianjin 300384, China
}

\begin{abstract}
The interaction between current slow wave and moving conductive medium is analyzed, and correspondingly a novel conversion mechanism of electromagnetic energy to continuous movement is proposed. The theoretical analysis and its experimental validation have shown the feasibility of the promising RF and microwave motors.
\end{abstract}

\section{Introduction}

The interconversion of electric and mechanical energies is always a fundamental problem in energy conversion. Motors and generators are its representative devices. It is well known that almost all motors and generators convert energy from one form to another through the action of a magnetic field. Usually the magnetic field is confined in a magnetic circuit, and the rotating magnetic field in the gap is created by currents from multiphase electric circuit system; both magnetic and electric circuits are operating at a very low frequency, and therefore can be considered independent of each other. The progresses of science and technology have introduced new demands in energy conversion, and one is related to converting $\mathrm{RF} /$ microwave energy to motion or kinetic energy. As early as in the sixties of last century, so called parametric motor or microwave motor [1-2] appeared. That motor was essentially a DC motor with a rectenna, which inserted inside the waveguide to pick up and rectify the microwave energy into DC energy then feed it to the motor coil [3]. Without direct conversion way, this indirect scheme has been continued until now in electric vehicles [4] and flights [5-6] etc. Nowadays, Solar Power Station/Satellite is a sustainable energy source to help meet the growing energy needs of developed and developing nations. One of the most important technologies for its realization is Wireless Power Transmission (WPT) [7]. The more using of WPT for the next generation will make the conversion mechanism from electromagnetic (EM) energy to other energies much more important. Unfortunately, the above mentioned fundamentals in electrical machinery become invalid at RF and microwave, because electric and magnetic fields are strongly coupled to each other, magnetic and electric circuits are mixed together, and RF and microwave multiphase system, in addition to the rotating magnetic or electric fields hardly can be feasible.

Own to the application demands, the explorations of the direct conversion mechanism from EM energy to kinetic energy have never ceased. Physical scientists had found that EM force, referred to radiation pressure in optical frequency band, can manipulate micro-objects [8] and even make micro-objects rotating [9], but probably it is still not big enough with the viewpoint of electric machinery. The effort has continued so far to enhance EM forces [10]; as a part of this effort, here we will report our research on the conversion mechanism of EM energy ( $\mathrm{RF} /$ microwave) to continuous movement, which may find variant applications in fields of Power and Energy Engineering.

\section{Conversion mechanisms}

The conversion mechanism of EM energy to continuous movement is based on the interaction between current slow wave and moving conductive medium. To analyze the interaction, we consider a simplified planar model of a motor driven by EM wave as shown in Figure 1. EM fields are excited by a surface current density in the stator at $z=0$, i.e.

$$
\boldsymbol{J}=\boldsymbol{y}_{0} J \exp [\mathrm{j}(\omega t-\alpha x)]
$$

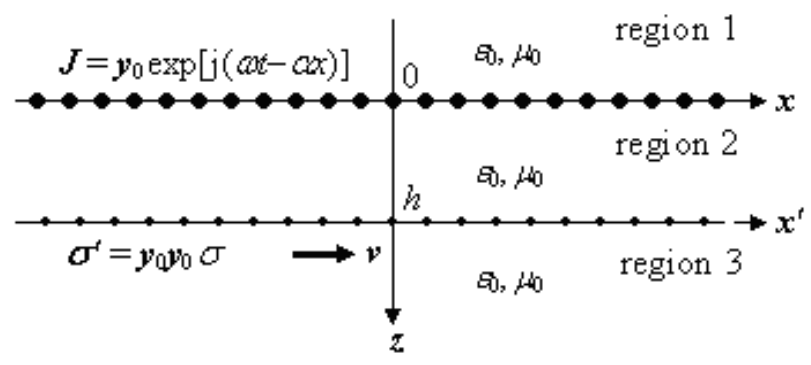

Figure 1. Current wave over a moving plate with dyad conductivity.

in which $J, \omega$ and $\alpha$ are the amplitude, the angular frequency and the propagation constant in $x$-direction, respectively. For a slow wave, $\alpha$ is greater than the propagation constant $k_{0}=\left(\omega^{2} \varepsilon_{0} \mu_{0}\right)^{1 / 2}$ in the air, and therefore the phase velocity in $x$-direction

\footnotetext{
a Corresponding author: xiadan@tjut.edu.cn, waiting831@163.com
} 


$$
v_{\mathrm{p}}=\frac{\omega}{\alpha}
$$

is less than the light speed $c=\omega / k_{0}[11]$. The rotor at $z=$ $h$ is a very thin conductive plate which is moving at the velocity $\boldsymbol{v}=\boldsymbol{x}_{0} v$, and its dyad surface conductivity represented in moving frame is

$$
\boldsymbol{\sigma}^{\prime}=\boldsymbol{y}_{0} \boldsymbol{y}_{0} \sigma
$$

All other regions are stationary free space with permittivity $\varepsilon_{0}$ and permeability $\mu_{0}$.

Electrical fields of slow TE ( $y$-polarized) wave in regions $i=1,2$, and 3 are expressed as

with

$$
\boldsymbol{E}_{i}=\boldsymbol{y}_{0} V_{i}(z) \mathrm{e}^{\mathrm{j}(\omega t-\alpha x)}
$$

$$
\begin{gathered}
V_{1}(z)=b_{1} \exp \left(\mathrm{j} k_{z} z\right) \\
V_{2}(z)=a_{2} \exp \left(-\mathrm{j} k_{z} z\right)+b_{2} \exp \left(\mathrm{j} k_{z} z\right) \\
V_{3}(z)=a_{3} \exp \left(-\mathrm{j} k_{z} z\right)
\end{gathered}
$$

where $b_{1}, a_{2}, b_{2}$, and $a_{3}$ are constants to be determined. The propagation constant in $z$-direction is

$$
k_{z}=\sqrt{\frac{\omega^{2}}{c^{2}}-\alpha^{2}}=-\mathrm{j} \gamma
$$

in which

$$
\gamma=\sqrt{\alpha^{2}-\frac{\omega^{2}}{c^{2}}}=\omega \sqrt{\frac{1}{v_{\mathrm{p}}^{2}}-\frac{1}{c^{2}}}
$$

is real.

By use of Maxwell's equation $\nabla \times \boldsymbol{E}=-\mu_{0} \partial \boldsymbol{H} / \partial t$, the corresponding $H$-fields can be derived as

$$
\boldsymbol{H}_{i}=\left[-\boldsymbol{x}_{0} I_{i}(z)+\boldsymbol{z}_{0} \frac{\alpha}{\omega \mu_{0}} V_{i}(z)\right] \mathrm{e}^{\mathrm{j}(\omega t-\alpha x)}
$$

with

$$
\begin{gathered}
I_{1}(z)=-Y b_{1} \exp \left(\mathrm{j} k_{z} z\right) \\
I_{2}(z)=Y\left[a_{2} \exp \left(-\mathrm{j} k_{z} z\right)-b_{2} \exp \left(\mathrm{j} k_{z} z\right)\right] \\
I_{3}(z)=Y a_{3} \exp \left(-\mathrm{j} k_{z} z\right)
\end{gathered}
$$

and mode admittance

$$
Y=\frac{k_{z}}{\omega \mu_{0}}=-\mathrm{j} \frac{\gamma}{\omega \mu_{0}}=-\mathrm{j} \frac{1}{\mu_{0}} \sqrt{\frac{1}{v_{\mathrm{p}}^{2}}-\frac{1}{c^{2}}}
$$

The boundary conditions at the interfaces $z=0$ and $z$ $=h$ are

$$
\begin{aligned}
& \boldsymbol{z}_{0} \times\left.\left(\boldsymbol{E}_{2}-\boldsymbol{E}_{1}\right)\right|_{z=0}=0 \\
& \boldsymbol{z}_{0} \times\left.\left(\boldsymbol{H}_{2}-\boldsymbol{H}_{1}\right)\right|_{z=0}=\boldsymbol{J} \\
& \boldsymbol{z}_{0} \times\left.\left(\boldsymbol{E}_{3}-\boldsymbol{E}_{2}\right)\right|_{z=h}=0 \\
& \boldsymbol{z}_{0} \times\left.\left(\boldsymbol{H}_{3}-\boldsymbol{H}_{2}\right)\right|_{z=h}=\boldsymbol{J}_{\mathrm{e}}
\end{aligned}
$$

where $\boldsymbol{J}_{\mathrm{e}}$ is the equivalent surface current density on the moving conductive plate. To count the contribution of the moving conductive plate, it is necessary to express the fields in moving frame. In the case of $v$ much smaller than $c$, Galilean transformations $x^{\prime}=x-v t, y^{\prime}=y$, $z^{\prime}=z$, and $t^{\prime}=t$ can be applied [12]. The fields in moving frame are

$$
\begin{aligned}
& \boldsymbol{E}_{i}{ }^{\prime}=\boldsymbol{E}_{i}+\boldsymbol{v} \times \mu_{0} \boldsymbol{H}_{i} \\
& \boldsymbol{H}_{i}{ }^{\prime}=\boldsymbol{H}_{i}-\boldsymbol{v} \times \varepsilon_{0} \boldsymbol{E}_{i}
\end{aligned}
$$

The conductive current can then obtain that

$$
\boldsymbol{J}_{\mathrm{e}}{ }^{\prime}=\left.\boldsymbol{\sigma}^{\prime} \cdot\left(\boldsymbol{E}_{3}{ }^{\prime}+\boldsymbol{E}_{2}{ }^{\prime}\right)\right|_{z^{\prime}=h}
$$

Both tangential (parallel to $v$ ) component of $H$-field and current density are invariant for Galilean transformations. Therefore, substituting the fields in stationary frame into Eqs. (7a) (7c) and those in moving frame into Eq. (7d), one can solve out the four unknown constants $b_{1}, a_{2}, b_{2}$, and $a_{3}$. Finally, we have

$$
V_{3}(h)=-\frac{J \exp (-\gamma h)}{2 \sigma\left(1-\frac{v}{v_{\mathrm{p}}}\right)-\mathrm{j} \frac{2}{\mu_{0}} \sqrt{\frac{1}{v_{\mathrm{p}}{ }^{2}}-\frac{1}{c^{2}}}}
$$

Nothing but the EM force density will make the rotor moving. It can be derived by use of $\boldsymbol{F}=\boldsymbol{J}_{\mathrm{e}}{ }^{\prime} \times \boldsymbol{B}_{3}{ }^{\prime}=$ $\boldsymbol{J}_{\mathrm{e}}{ }^{\prime} \times \mu_{0} \boldsymbol{H}_{3}{ }^{\prime}$ and represented in terms of $V_{3}(h)$.

\section{Performances}

Suppose that the rotor is only possible to move in $x$ direction. Thus the $x$-component of EM force density $F_{x}$ is most concerned, and it is related to the $z$-component of flux density

$$
B_{3 z}{ }^{\prime}=\left(\frac{1}{v_{\mathrm{p}}}-\frac{v}{c^{2}}\right) V_{3}(h) \mathrm{e}^{\mathrm{j}(\omega t-\alpha x)}
$$

and the $y$-component of current density

$$
J_{\mathrm{e}}^{\prime}=2 \sigma\left(1-\frac{v}{v_{\mathrm{p}}}\right) V_{3}(h) \mathrm{e}^{\mathrm{j}(\omega t-\alpha x)}
$$

To keep continuous movement of rotor in $x$-direction, it is required that the effective (average) force density in $x$-direction $\left\langle F_{x}\right\rangle$ is non-zero. $\left\langle F_{x}\right\rangle$ can be obtained by integrating the EM force density over space period $2 \pi / \alpha$ and time period $2 \pi / \omega$, respectively, and the result is

$$
\left\langle F_{x}\right\rangle=\frac{1}{2} \operatorname{Re}\left(J_{\mathrm{e}}{ }^{\prime} B_{3 z}{ }^{\prime *}\right)
$$

where * denotes complex conjugate. Substituting Eq. (11) into Eq. (12), it follows that

$$
\left\langle F_{x}\right\rangle=\frac{J^{2} \sigma\left(1-\frac{v}{v_{\mathrm{p}}}\right)\left(\frac{1}{v_{\mathrm{p}}}-\frac{v}{c^{2}}\right)}{4 \sigma^{2}\left(1-\frac{v}{v_{\mathrm{p}}}\right)^{2}+\frac{1}{\mu_{0}^{2}}\left(\frac{1}{v_{\mathrm{p}}^{2}}-\frac{1}{c^{2}}\right)} \mathrm{e}^{-2 \gamma h}
$$

Letting $v=0$ in Eq. (13), the starting force is expressed as 


$$
\left\langle F_{x}\right\rangle_{\mathrm{s}}=\frac{1}{4} \frac{J^{2} \sigma \frac{1}{v_{\mathrm{p}}}}{\sigma^{2}+\frac{1}{\mu_{0}^{2}}\left(\frac{1}{v_{\mathrm{p}}{ }^{2}}-\frac{1}{c^{2}}\right)} \mathrm{e}^{-2 \gamma h}
$$

It is worth noting that the starting force is depended on the factors: $v_{\mathrm{p}}^{-1}, \sigma\left[\sigma^{2}+\left(v_{\mathrm{p}}{ }^{-2}-c^{-2}\right) / \mu_{0}^{2}\right]^{-1} \approx \sigma^{-1}$ and $J^{2} \exp (-2 \gamma h)$. It implies that in order to obtain the larger starting force, we must make the phase velocity of EM wave, the surface conductivity and the air gap smaller, and in turn the amplitude of current source $J$ larger. Taken typical parameters of phase velocity in $x$-direction $v_{\mathrm{p}}=0.01 c$ and unit current $J=1$, the force-speed curves with different values of surface conductivity and relative thickness of air gap $h / \lambda, \lambda=c / f$ is the wavelength in the gap, are calculated by using Eq. (13) and shown in Figure 2.

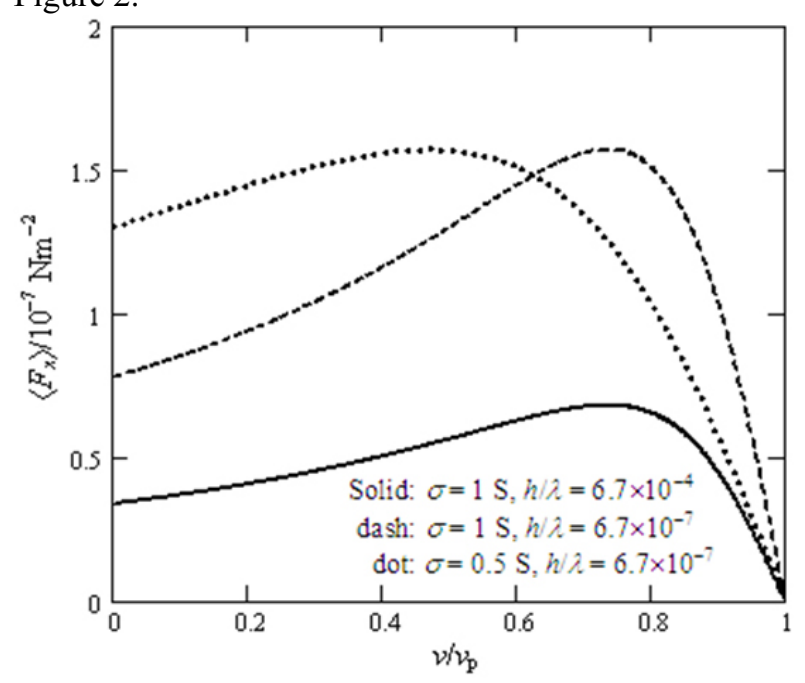

Figure 2. Force-speed curves.

It is obvious from the curves that the force is larger for smaller relative thickness of air gap. The other behaviors of the model are somewhat similar to the traditional induction motor. At synchronous speed $v=v_{\mathrm{p}}$, the force is always vanished; at certain speed that is near but different from synchronous speed $v_{\mathrm{p}}$, the force gets the maximum value, and less conductivity $\sigma$ leads to larger starting force and bigger speed slip $\left(v_{\mathrm{p}}-v\right)$ for the maximum force.

\section{Experimental validation}

The experiment has two principal aims: to test if the above conversion mechanism really works and to estimate the starting force under the excitation. The prototype used in the experiment is shown in Figure 3. Here the moving part (rotor) is a conductive film on a disk-shaped substrate (Figure 3 right), while a circular array of slots are etched on the film to make the film anisotropic with the conductivity as defined by Eq. (2). The stationary part (Figure 3 left) can be regarded as a converter from high frequency voltage to slow wave, and it is designed with phase velocity $v_{\mathrm{p}} \approx 0.01 c$ and wave impedance $Z_{0} \approx 75 \Omega$.

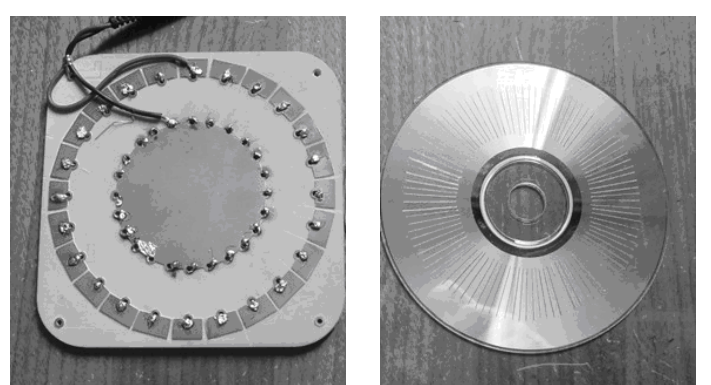

Figure 3. Prototype stator (left) and rotor (right).

In the experiment, the moving part is assembled as a torsion pendulum [13] so that the possible rotation can be observed and the toque can be estimated when it is in balance after rotating. The voltage-to-wave converter is excited by a high frequency signal generator at the starting port and matched with a $75 \Omega$ load at the end port The travelling slow wave as defined by Eq. (3) is incident on the surface of the rotor and the EM force density in $x$-direction $\left\langle F_{x}\right\rangle$ makes it moving. Its movement can be observed in full frequency range from 0.150 to $1 \mathrm{GHz}$ of the signal generator. The measured deviation angles $\theta$ of the torsion pendulum at different frequencies $f$ are listed in Table 1 .

Table 1. Deviation angles $\theta$ at different frequencies.

\begin{tabular}{|c|c|c|c|c|c|}
\hline$f / \mathrm{MHz}$ & 150 & 266 & 290 & 340 & 440 \\
\hline$\theta /$ degree & -15 & -11.3 & 15 & 33.3 & 37.5 \\
\hline$f / \mathrm{MHz}$ & 540 & 650 & 750 & 850 & 1000 \\
\hline$\theta /$ degree & 37.5 & 37.5 & 37.5 & 56.3 & 41.3 \\
\hline
\end{tabular}

The deviation angle can roughly reflect the amplitude of the starting force. The positive and negative angles in the table tell us an unusual fact that the forces can have different directions in different frequency bands. This interesting fact can be understood by invoking the particular frequency characteristics of the voltage-to-wave converter. Taking the stray parameters into account, the converter can be modeled as a composite right/left-handed transmission line [14], whose unitary cell and phase velocity-to-frequency curve [15] are shown in Figure 4. Here $L_{R}$ and $C_{R}$ are stray parameters, and $L_{L}$ and $C_{L}$ are real parameters. One can figure out from the curve that the phase velocity can change its direction near the resonant frequency, so can the starting force in the experiment [16].

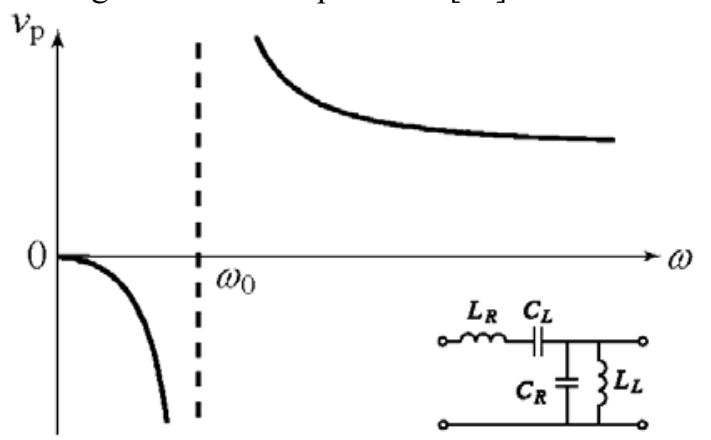

Figure 4. Unitary cell (lower right) and phase velocity-tofrequency curve of composite right/left-handed transmission line. 


\section{Conclusions}

The proposed conversion mechanism of EM energy to continuous movement in this paper is based on the interaction between current slow wave and moving conductive medium. Invoking the novel exciting scheme of slow EM wave, the model of motor is successfully to operate at RF/microwave frequencies. To the best of our knowledge, it is the first time to make a macroscopic object into continuous moving state directly by $\mathrm{RF} /$ microwave EM force. Although the experiment of prototype is rather primary, the analysis based on rigorous EM theory and its experimental validation have provided sound base for further study on practical device of such a kind of motors, which may find a series of potential applications in the fields of Power and Energy Engineering, such as directly wireless energy storage by flywheel and wireless power distribution to mobile users. Moreover, the applications in other fields are also possible.

\section{References}

1. H.E. Stockman, Proceedings of the IEEE, 51, 12531254 (1963)

2. R.C. Garnier, K. Ishii, Proceedings of the IEEE, 52, 1380-1381 (1964)

3. R.W. Steinhoff, T.K. Ishii, IEEE T. Microw. Theory, 14, 438-438 (1966)

4. A. Oida, H. Nakashima, J. Miyasaka, K. Ohdoi, H. Matsumoto, N. Shinohara, J. Terramechanics, 44, 329-338 (2007)

5. J.J. Schlesak, A. Alden, T. Ohno, IEEE MTT-S, 1, 283-286 (1988)

6. E. Shimane, S. Komatsu, K. Komurasaki, Y. Arakawa, $46^{\text {th }}$ AIAA, 2008-1149

7. S.S. Mohammed, K. Ramasamy, T. Shanmuganantham, IJCA, 13, 100-103 (2007)

8. A. Ashkin, IEEE J. Sel. Top. Quant., 6, 841-856 (2000)

9. E. Higurashi, O. Ohguchi, T. Tamamura, H. Ukita, R. Sawada, J. Appl. Phys., 82, 2773-2779 (1997)

10. A. Mizrahi, M. Horowitz, L. Schächter, Phys. Rev. A, 78, 023802 (2008)

11. D. Xia, T.L. Dong, Chinese Sci. Bull., 55, $3875-$ 3879 (2010)

12. J.A. Kong, Electromagnetic Wave Theory (EMW Publishing, Cambridge, Massachusetts, 2000)

13. V.B. Braginsky, A.B. Manukin, G.M. Keiser, Phys. Today, 31, 195-196 (1978)

14. T. Günel, AEÜ, 61, 459-462 (2007)

15. C. Caloz, T. Itoh, Electromagnetic Metamaterials: Transmission Line Theory and Microwave Applications: The Engineering Approach (John Wiley \& Sons, Inc., Hoboken, New Jersey, 2006)

16. D. Xia, R.X. Yao, P. Li, G. Feng, T.L. Dong, APPEEC 2011 